Lindomar Jacinto de Souza

Adequação das Ações de Controle de Tensão em Sistemas Elétricos Objetivando o Reforço das Condições de Segurança de Tensão

Tese de Doutorado

Tese apresentada como requisito parcial para obtenção do título de Doutor pelo Programa de Pós-Graduação em Engenharia Elétrica da PUC-Rio.

Orientador: Ricardo B. Prada 
Lindomar Jacinto de Souza

\section{Adequação das Ações de Controle de Tensão em Sistemas Elétricos Objetivando o Reforço das Condições de Segurança de Tensão}

Tese de Doutorado apresentada como requisito parcial para obtenção do grau de Doutor pelo Programa de PósGraduação em Engenharia Elétrica do Departamento de Engenharia Elétrica do Centro Técnico Científico da PUC-Rio. Aprovada pela Comissão Examinadora abaixo assinada.

Dr. Ricardo B. Prada

Orientador

Departamento de Engenharia Elétrica - PUC-Rio

Dr. João Passos Filho

CEPEL

Dr. Glauco Taranto

COPPE / UFRJ

Dr. José Eduardo Onoda Pessanha

UFMA

Dr. Jorgeson O. R. dos Santos

UFSJ

Dr. Carlos A. Castro

UNICAMP

Prof. José Eugenio Leal

Coordenador Setorial do Centro

Técnico Científico - PUC-Rio

Rio de Janeiro, 30 de março de 2007 
Todos os direitos reservados. É proibida a reprodução total ou parcial do trabalho sem autorização da universidade, do autor e do orientador.

\section{Lindomar Jacinto de Souza}

Graduado em Engenharia Elétrica pela Universidade Federal do Maranhão (UFMA), em 1995. Mestre em Engenharia Elétrica pela UFMA em 1997. É professor Adjunto do Departamento de Eletroeletrônica do Centro Federal de Educação Tecnológica do Maranhão (CEFETMA), desde 1998.

Ficha Catalográfica

Souza, Lindomar Jacinto de

Adequação das ações de controle de tensão em sistemas elétricos objetivando o reforço das condições de segurança de tensão / Lindomar Jacinto de Souza; orientador: Ricardo B. Prada. - Rio de Janeiro: PUCRio, Departamento de Engenharia Elétrica, 2007.

203 f. : il.; $30 \mathrm{~cm}$

Tese (doutorado) - Pontifícia Universidade Católica do Rio de Janeiro, Departamento de Engenharia Elétrica.

Inclui referências bibliográficas.

1. Engenharia elétrica - Teses. 2. Controle de tensão. 3. Estabilidade de tensão. 4. Segurança de tensão. 5. Colapso de tensão. 6. Análise de autovalores. I. Prada, Ricardo B. II. Pontifícia Universidade Católica do Rio de Janeiro. Departamento de Engenharia Elétrica. III. Título. 
A Deus,

meus pais, Ângelo e Dalva, e irmãos, Delcimar, Ângela e Lucimar, pelo apoio e confiança. 


\section{Agradecimentos}

Ao Professor Ricardo Prada, pela dedicação e orientação segura, imprescindível para o desenvolvimento deste trabalho, e pelo apoio prestado durante todo doutoramento.

À FAPEMA pelo suporte financeiro concedido.

Ao CEPEL pela licença de uso dos programas computacionais ANAREDE e FLUPOT.

À PUC-Rio pela concessão de bolsa de isenção de pagamento de mensalidades, sem a qual não poderia fazer o doutoramento, e aos funcionários do seu Departamento de Engenharia Elétrica, por toda atenção prestada.

À Karla Keissan, que esteve do meu lado torcendo pelo meu sucesso.

Aos professores que participaram da comissão examinadora.

Aos professores Osvaldo Saavedra, Maria da Guia, e tantos outros do Departamento de Engenharia Elétrica da UFMA, pelo incentivo prestado desde o princípio de minha vida acadêmica.

A todos os parceiros do curso de Pós-Graduação em Engenharia Elétrica da PUC-Rio pelo estímulo e ajuda no decorrer do curso, em especial ao amigo David Paco.

Por fim, a Deus novamente, por ter me colocado em uma família maravilhosa... 


\section{Resumo}

Souza, Lindomar Jacinto de; Prada, Ricardo B. Adequação das Ações de Controle de Tensão em Sistemas Elétricos Objetivando o Reforço das Condições de Segurança de Tensão. Rio de Janeiro, 2007. 203p. Tese de Doutorado - Departamento de Engenharia Elétrica, Pontifícia Universidade Católica do Rio de Janeiro.

O problema de estabilidade de tensão está intimamente ligado à falta de recursos e questões ambientais que limitam a expansão do sistema de transmissão. Esta realidade em conjunto com o crescimento da carga submete os sistemas elétricos a carregamentos pesados, podendo levar a situações de colapso de tensão. O desenvolvimento de métodos para avaliação do carregamento da rede de transmissão tornou-se necessário e imprescindível para que se possa entender o funcionamento do sistema nestas condições e possibilitar a sua operação de modo correto. Em relatório de força-tarefa internacional está explicitada a necessidade de uma ferramenta computacional capaz de verificar a eficácia das ações de controle de tensão na operação do sistema elétrico em tempo-real. Com esse objetivo, foi desenvolvida uma ferramenta computacional com base no sistema linearizado das equações de fluxo de carga, e de todas as outras equações de controles e limites, julgadas pertinentes, para avaliação do efeito das ações de controle de tensão. Determinase uma matriz de sensibilidade [VCS], "voltage control sensitivity matrix", através da qual se pode estabelecer a relação existente entre as tensões controladas e as grandezas controladoras. Os elementos diagonais relacionam a grandeza controladora de cada equipamento com a respectiva tensão controlada. A análise do sinal desses elementos estabelece se uma determinada ação de controle será adequada ou não, isto é, se terá efeito esperado ou oposto. Os elementos fora da diagonal representam a interdependência existente entre os equipamentos controladores de tensão. A matriz de sensibilidade também permite que ações de controle de tensão sejam calculadas com o objetivo de "mover" um ponto de operação inadequado, ou que esteja suscetível à inadequacidade do controle de tensão, colocando-os em um ponto de operação seguro, onde ações operativas de controle de tensão tenham o efeito esperado. Os resultados da avaliação do 
método desenvolvido e aplicado a sistemas-teste e reais foram qualitativamente coerentes com aqueles obtidos pela resolução (analítica, iterativa por Newton ou iterativa continuada) das equações de fluxo de carga, validando a proposta. A dimensão da matriz [VCS] é proporcional ao número de barras com tensão controlada em análise. Esta matriz não é esparsa e então, quando utilizada para representar o controle de tensão realizado por um número elevado de equipamentos controladores, sua análise direta pode se tornar complexa. Portanto, uma análise baseada nos autovalores e autovetores associados à matriz [VCS] é aplicada objetivando identificar barras de tensão controlada com efeito reverso, ocorrendo de modo contrário ao esperado, e a existência de controles conflitantes. Buscam-se autovalores negativos, nulos ou próximos de zero. A localização das barras com problemas se dá através do exame dos autovetores e fatores de participação associados a esses autovalores. O cálculo e a análise da matriz de sensibilidade dos controles de tensão são executados de forma extremamente rápida e, portanto, o esforço computacional não impede que a ferramenta desenvolvida possa ser usada durante a operação em tempo-real.

\section{Palavras - chave}

Controle de tensão, adequação do controle de tensão, segurança de tensão, colapso de tensão, análise de autovalores. 


\section{Abstract}

Souza, Lindomar Jacinto de; Prada, Ricardo B. Voltage Control Sensitivity Calculation for Voltage Stability Operational Reinforcement. Rio de Janeiro, 2007. 203p. Doctorate Thesis - Electrical Engineering Department, Pontifícia Universidade Católica do Rio de Janeiro.

The voltage stability problem has been associated with environment questions and lack of financial resources for transmission system expansion. This reality together with the load growth makes the electrical system to be subject of heavy loadings, what can lead to situations of voltage collapse. The development of methods for evaluation of transmission network loading became necessary and indispensable in order to correct operation of the system under heavy loading conditions. In an international force-task report it is stated the need for analytical tools capable of verifying the voltage control action adequacy in the real-time operation. This work reports the development of a computation tool able to evaluate the effect of voltage control actions on the voltage itself. It is based the linearized load flow equations, including control and limit equations judged pertinent. The tool establishes a sensitive matrix, named [VCS] for "voltage control sensitivity matrix", that relates the controlling variables and the controlled voltages. Through the analysis of the sign of each diagonal element, it is possible to know whether a specific control action is adequate or not. Moreover, the off-diagonal elements represent the interdependence among the voltage controller devices of the power system under analysis. The sensitivity matrix also allows the calculation of control actions necessary to "move" the operating point from an unstable area, or near by, to a secure operating region where all voltage control actions would have the expected effect. The results obtained with the "voltage control sensitivity matrix" applied on real and test-systems was qualitatively coherent with those calculated by analytical, iteractive by Newton-Raphson and/or continuationiteractive solution of power flow equations, validating the proposed method. The [VCS] dimension is proportional to the number of buses with controlled voltage in the system area under analysis. The matrix is not sparse and when there are a large number of voltage control equipments, its immediate analysis may be complex. Therefore, an analysis based on eigenvalues and eigenvectors 
associated with [VCS] matrix is used in order to identify voltage control action with opposite effect. In this case, the analysis is focused on negative eigenvalues. The identification of conflicting controls existence of any system area under analysis is a by-product. The "voltage control sensitivity matrix" computation and analysis are performed extremely fast. Therefore, the computational effort does not constrain its use in real-time operation.

\section{Keywords}

Voltage Control, voltage control sensitivity, voltage security, voltage stability, voltage collapse, eigenvalue analysis. 


\section{Sumário}

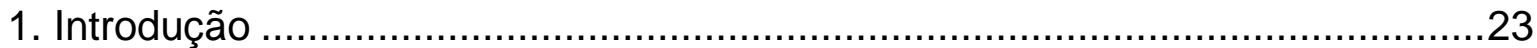

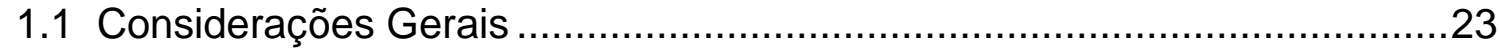

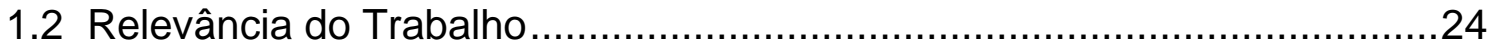

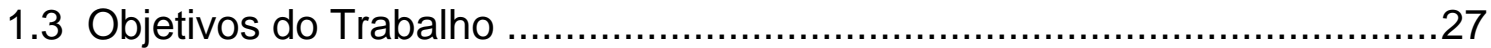

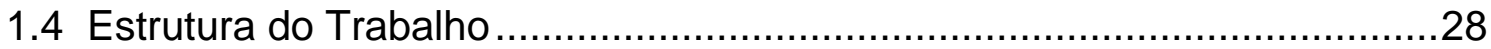

2. Avaliação e Reforço das Condições de Estabilidade de Tensão .....................29

2.1 Avaliação Nodal do Carregamento da Rede de Transmissão ...................29

2.1.1 Método de Análise ...........................................................29

2.1.1.1 Magnitude do Determinante da Matriz $\left[D^{\prime}\right]$.......................31

2.1.1.2 Sinal do Determinante da Matriz [D] ..............................34

2.1.1.3 Interpretação dos Índices ............................................. 36

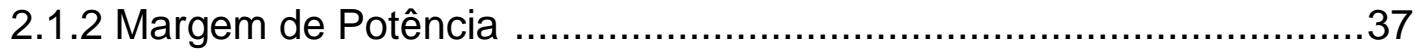

2.1.3 Índice de Influência ............................................................. 38

2.2 Determinação de Ações de Controle ......................................................40

2.2.1 Transformação do Sistema Multi-nó ...........................................40

2.2.2 Reforço em Barras com Tensão Controlada..................................43

2.2.2.1 Reforço em Barras de Geração .......................................44

2.2.2.2 Reforço em Barras com Compensação Reativa...................44

2.2.3 Aplicação em um Sistema-Teste de 5 Barras................................46

2.2.3.1 Aplicação do Programa EstabTen ...................................48

2.2.3.2 Aplicação do Programa CaTrans.....................................48

2.2.3.3 Aplicação do Programa FLUPOT ....................................49

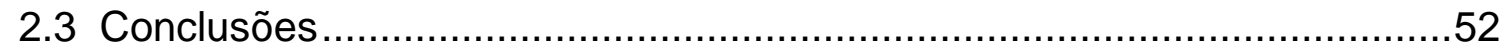

3. Adequação das Ações de Controle de Tensão .....................................53

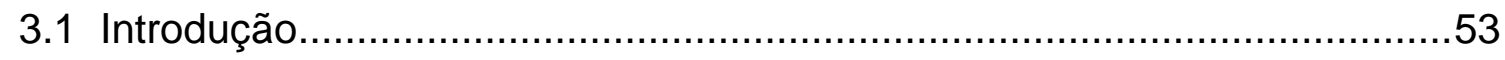

3.2 Representação dos Controles de Tensão e Cálculo dos Índices ...............53

3.3 Controle de Tensão por Gerador e Compensador Síncrono .......................54 
3.3.1 Índice Tensão Interna vs. Tensão Terminal.....................................55

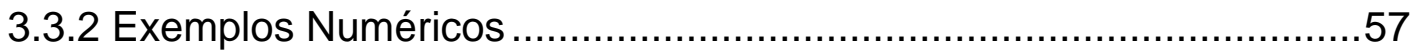

3.3.2.1 Índice Tensão Interna vs. Tensão Terminal para Controle Local.

3.3.2.2 Índice Tensão Interna vs. Tensão Terminal para

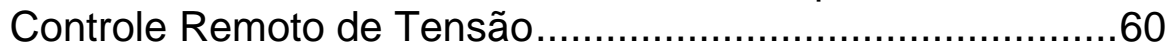

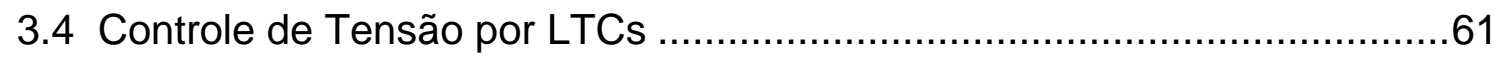

3.4.1 Índice Tape vs. Tensão da Barra Controlada ..................................61

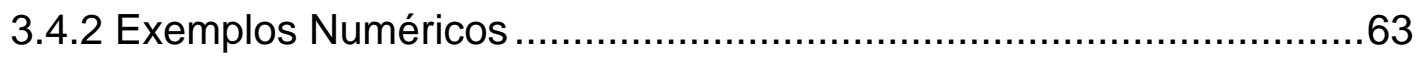

3.4.2.1 Índice Tape do LTC vs. Tensão da Barra

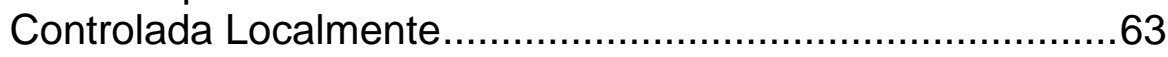

3.4.2.2 Índice Tape do LTC vs. Tensão da Barra

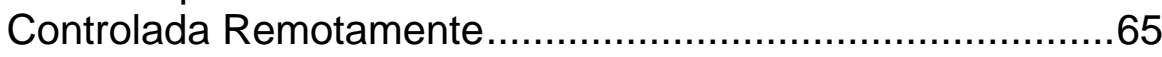

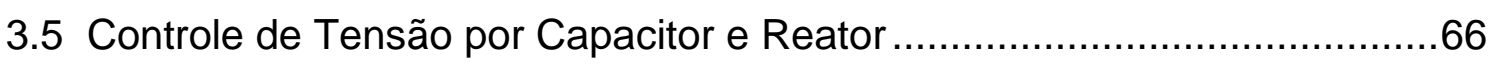

3.5.1 Índice Susceptância vs. Tensão Terminal .......................................66

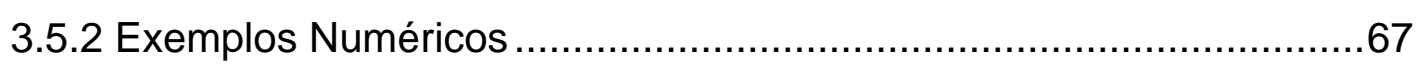

3.5.2.1 Índice Susceptância vs. Tensão Terminal ...........................67

3.5.2.2 Índice Susceptância vs. Tensão Remota...............................69

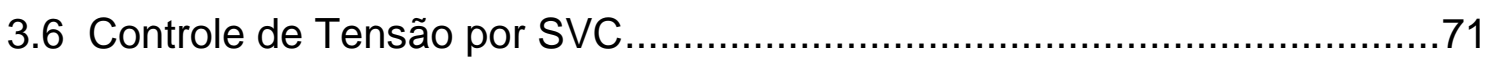

3.6.1 Índice Susceptância vs. Tensão Terminal ......................................72

3.6.2 Índice Ângulo de Disparo vs. Tensão Terminal ................................73

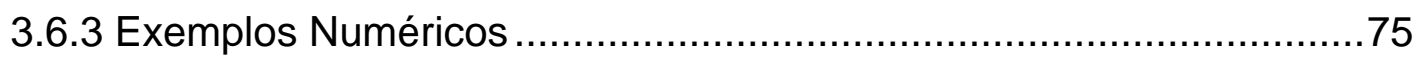

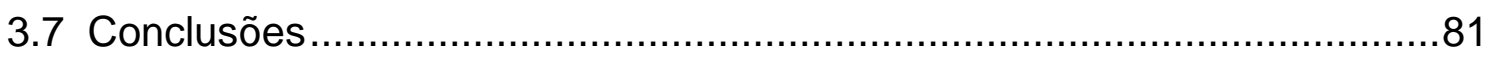

4. Matriz de Sensibilidade dos Controles de Tensão ........................................82

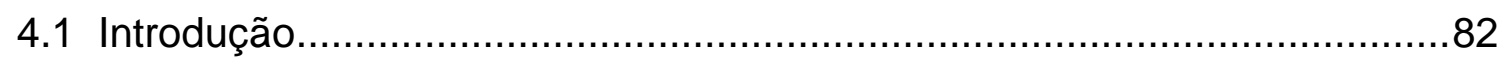

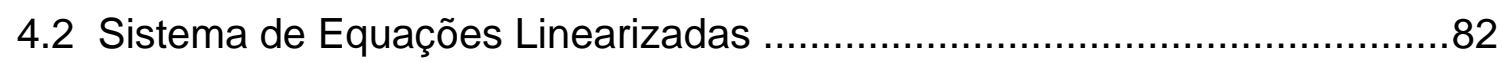

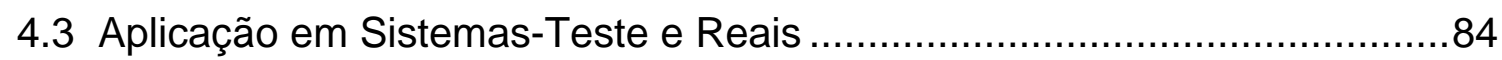

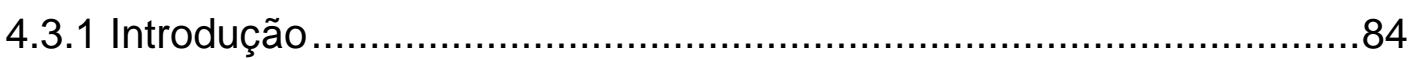

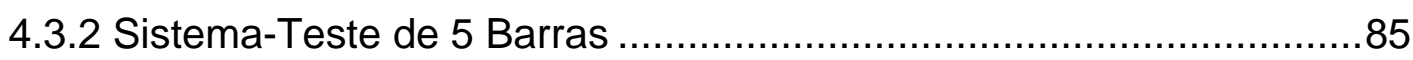

4.3.3 Interação Entre os Equipamentos Controladores de Tensão ............95

4.3.4 Esforço Computacional para Sistemas de Grande Porte.................101

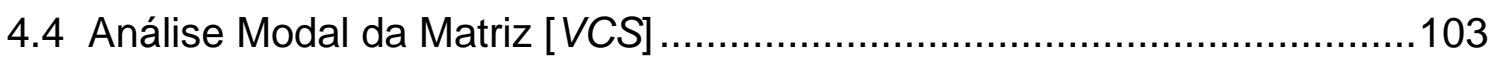

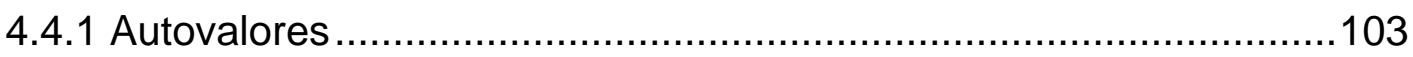

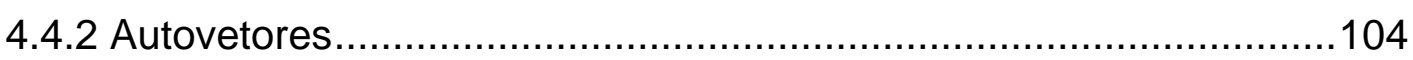

4.5 Fator de Participação de Equipamentos Controladores de Tensão .........105 


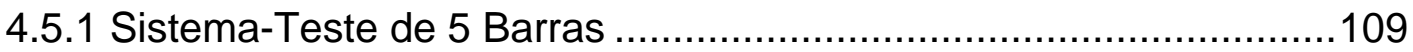

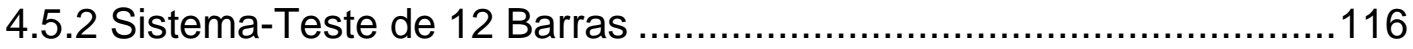

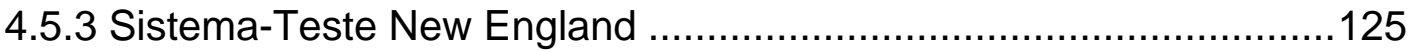

4.5.4 Avaliação do Sistema-Teste SAGE 35 Barras ................................141

4.5.5 Avaliação do Sistema S-SE Brasileiro …………………….........149

4.5.6 Avaliação do Sistema Sul, ONS, Curto Prazo, Dez / 99 ...................157

4.6 Eficiência Computacional ...............................................................

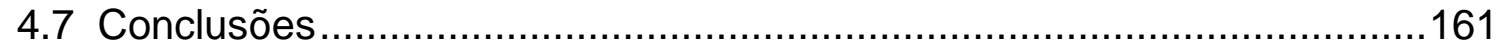

5. Obtenção do Ponto de Operação a Partir do Novo Perfil de Tensão ...............163

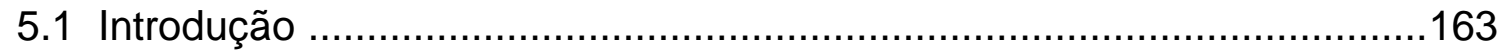

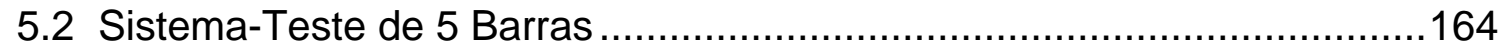

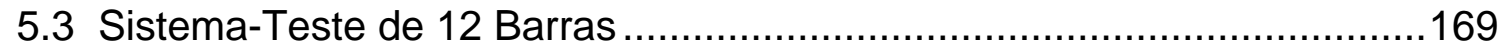

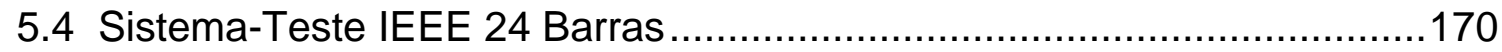

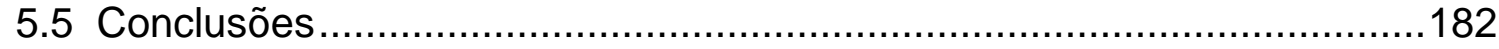

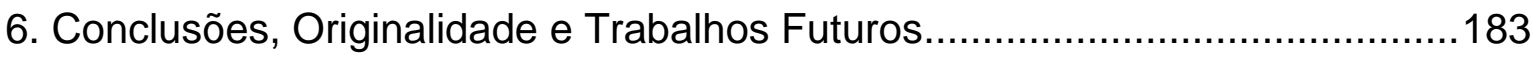

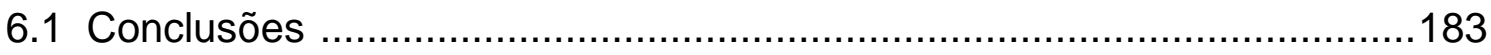

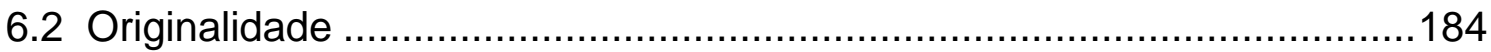

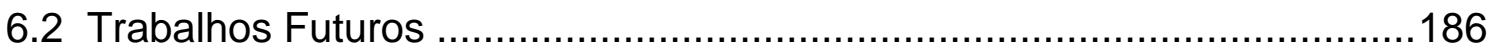

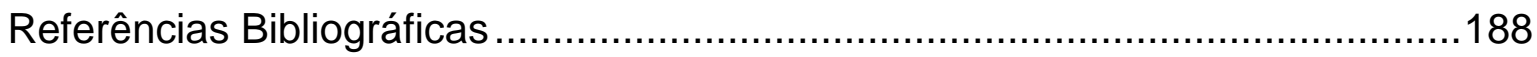

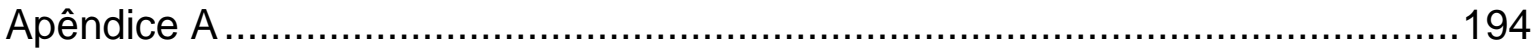

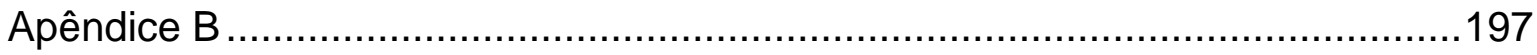




\section{Lista de Figuras}

Figura 2.1 - Localização dos Vetores Gradientes de $P_{i}$ e $Q_{i}$ no Plano V $\theta$.............35

Figura 2.2 - Sinal da Margem na Curva SV ................................................ 38

Figura 2.3 - Movimento dos Pontos de Operação na Curva SV ..........................39

Figura 2.4 - Curva SV para Análise do Índice de Influência ..............................39

Figura 2.5 - Curvas VQ para a Barra de Grajaú 500 kV ..................................45

Figura 2.6 - Diagrama Unifilar do Sistema-Teste de 5 Barras ..........................46

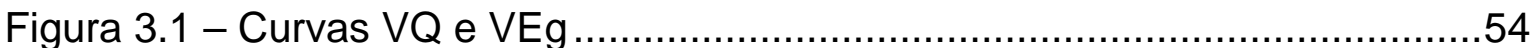

Figura 3.2 - Diagrama Unifilar do Modelo de Regime Permanente do Gerador ou Compensador Síncrono.

Figura 3.3 - Diagrama Unifilar de um Sistema com Gerador, Linha de Transmissão e Carga ...........................................................5 57

Figura 3.4 - Tensão ao Longo da Linha Desde a Barra g até a Barra 1 ...............57

Figura 3.5 - Tensões ao Longo da Rede Antes e Depois da Ação de Controle ...59

Figura 3.6 - Diagrama Unifilar de um LTC do Sistema

Figura 3.7 - Diagrama Unifilar do Sistema com Gerador, LTC, Linha de Transmissão e Carga

Figura 3.8 - Diagrama Unifilar do Sistema de 5 Barras .67

Figura 3.9 - Diagrama Unifilar do Sistema de 3 Barras 69

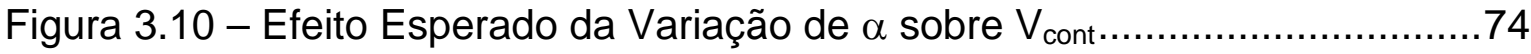

Figura 3.11 - Efeito Oposto ao Esperado da Variação de $\alpha$ sobre $V_{\text {cont }}$................75

Figura 3.12 - Diagrama Unifilar do Sistema de 5 Barras com SVC .75

Figura 3.13 - Susceptâncias do FC, TCR e SVC em Função do Ângulo de Disparo dos Tiristores

Figura 4.1 - Diagrama Unifilar do Sistema-Teste de 5 Barras .85

Figura 4.2 - Diagrama Unifilar do Sistema-Teste de 5 Barras Incluindo o Modelo dos Geradores

Figura 4.3 - Mode-Shape de $\lambda_{3}$ da Matriz de (4.17)

Figura 4.4 - Fator de Participação Calculado para $\lambda_{3}$ da Matriz de (4.17) ..........112

Figura 4.5 - Fator de Participação Calculado para $\lambda_{3}$ da Matriz de (4.19) ..........113

Figura 4.6 - Mode-Shape de $\lambda_{3}$ da Matriz de (4.19)

Figura 4.7 - Fator de Participação Calculado para $\lambda_{5}$ da Matriz de (4.28) ..........114

Figura 4.8 - Mode-Shape de $\lambda_{5}$ da Matriz de (4.28) ....................................114

Figura 4.9 - Fator de Participação Calculado para $\lambda_{5}$ da Matriz de (4.30) ..........115 
Figura 4.10 - Mode-Shape de $\lambda_{5}$ da Matriz de (4.30) 115

Figura 4.11 - Diagrama Unifilar do Sistema-Teste de 12 Barras 116

Figura 4.12 - Mode-Shape de $\lambda_{1}$ da Matriz de (4.57)...................................118

Figura 4.13 - Mode-Shape de $\lambda_{2}$ da Matriz de (4.57)...................................119

Figura 4.14 - Mode-Shape de $\lambda_{3}$ da Matriz de (4.57)....................................119

Figura 4.15 - Autovalores do Sistema-Teste de 12 Barras vs. Aumento Percentual da Carga

Figura 4.16 - Fatores de Participação do Autovalor $\lambda_{5}$ da Matriz de (4.58) ........124

Figura 4.17 - Mode-Shape do Autovalor $\lambda_{5}$ da Matriz de (4.58) .......................125

Figura 4.18 - Diagrama Unifilar do Sistema-Teste New England ......................126

Figura 4.19 - Fatores de Participação do Autovalor $\lambda_{4}=0,2711$ da Matriz [VCS] de (4.59) .....

Figura 4.20 - Mode-Shape do Autovalor $\lambda_{4}=0,2711$ da Matriz [VCS] de (4.59) 130

Figura 4.21 - Fatores de Participação do Autovalor $\lambda_{11}$ da Matriz [VCS] de (4.60) 134

Figura 4.22 - Mode-Shape do Autovalor $\lambda_{11}$ da Matriz [VCS] de (4.60). 134

Figura 4.23 - Fatores de Participação do Autovalor $\lambda_{12}$ da Matriz [VCS] de (4.60)

Figura 4.24 - Mode-Shape do Autovalor $\lambda_{12}$ da Matriz [VCS] de (4.60). 135

Figura 4.25 - Fatores de Participação do Autovalor $\lambda_{14}=0,2104$ da Matriz [VCS] de (4.61)

Figura 4.26 - Mode-Shape do Autovalor $\lambda_{14}=0,2104$ da Matriz [VCS] de (4.61)

Figura 4.27 - Diagrama Unifilar do Sistema-Teste SAGE 35 Barras

Figura 4.28 - Fatores de Participação do Autovalor $\lambda_{9}=-0,0154$ da Matriz [VCS] de (4.62)

Figura 4.29 - Mode-Shape do Autovalor $\lambda_{9}=-0,0154$ da Matriz [VCS] de (4.62)

Figura 4.30 - Diagrama Unifilar Simplificado do Sistema Sul 158

Figura 5.1 - Diagrama Unifilar do Sistema-Teste de 5 Barras com a Inclusão do Modelo de Regime Permanente....

Figura 5.2 - Relação Entre a Tensão Interna e a Tensão Terminal do Gerador 1

Figura 5.3 - Relação Entre a Tensão Interna e a Tensão Terminal do Gerador 2

Figura 5.4 - Diagrama Unifilar do Sistema-Teste IEEE 24 Barras 170 
Figura 5.5 - Fator de Participação Calculado para o Autovalor $\lambda_{8}$ da Matriz de [VCS] Dada em (5.6) .............................................................180

Figura 5.6 - Mode-Shape do Autovalor $\lambda_{8}$ da Matriz de [VCS] Dada em (5.6)...180

Figura 5.7 - Fator de Participação Calculado para o Autovalor $\lambda_{9}$ da Matriz de

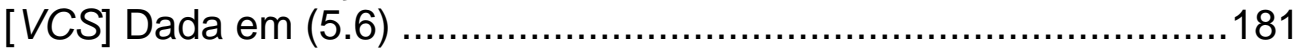

Figura 5.8 - Mode-Shape do Autovalor $\lambda_{9}$ da Matriz de [VCS] Dada em (5.6)....181 


\section{Lista de Tabelas}

Tabela 2.1 - Dados de Barra e de Linha do Sistema-Teste de 5 Barras .............46

Tabela 2.2 - Geração e Carga / Caso-Base .................................................. 47

Tabela 2.3 - Fluxo de Potência nas Linhas / Caso-Base ..................................47

Tabela 2.4 - Índices de Avaliação da Estabilidade / Caso-Base..........................48

Tabela 2.5 - Caminhos Associados à Barra de Carga / Caso-Base ....................49

Tabela 2.6 - Geração e Carga / Caso "Mínima Transferência de Potência"

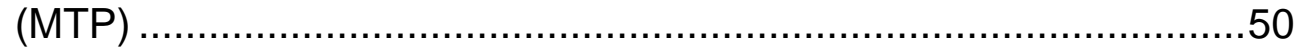

Tabela 2.7 - Fluxo nas Linhas / Caso "Mínima Transferência de Potência" .........50

Tabela 2.8 - Índices de Avaliação da Estabilidade / Caso "Mínima Transferência de Potência".

Tabela 2.9 - Caminhos Associados à Barra de Carga / Caso "Mínima Transferência de Potência"...

Tabela 3.1 - Ponto de Operação Onde a Barra 0 Está à Direita do Ponto P ........58

Tabela 3.2 - Ponto de Operação Após Decréscimo na Tensão Interna $E_{g} \ldots . . . . . .59$

Tabela 3.3 - Ponto de Operação Onde a Barra 0 Está à Esquerda do Ponto P ...59

Tabela 3.4 - Ponto de Operação Onde a Barra Controlada Está na Região

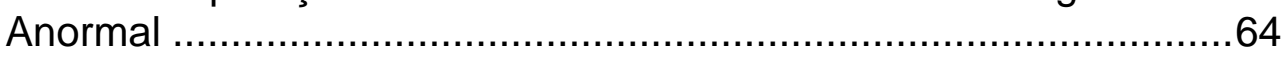

Tabela 3.5 - Ponto de Operação Onde a Barra Controlada Está na Região Normal ............................................................................64

Tabela 3.6 - Ponto de Operação com a Barra 5 na Região Normal ....................68

Tabela 3.7 - Ponto de Operação com a Barra 5 na Região Anormal ....................68

Tabela 3.8 - Ponto de Operação na Região Anormal ......................................70

Tabela 3.9 - Ponto de Operação na Região Normal.....................................70

Tabela 3.10 - Efeito do Aumento de $V_{\text {esp }}$ no Ponto de Operação da Região Normal .........................................................................

Tabela 3.11 - Efeito do Aumento de $V_{\text {esp }}$ no Ponto de Operação da Região Anormal............................................................................ 78

Tabela 4.1 - Dados do Sistema-Teste de 5 Barras........................................ 87

Tabela 4.2 - Dados do Sistema-Teste de 5 Barras / Região B .........................88

Tabela 4.3 - Equipamentos de Controle de Tensão com Efeito Oposto na Tensão da Barra Controlada i para [VCS] de (4.19).

Tabela 4.4 - Barras de Tensão Controlada Afetadas de Forma Oposta pelo Equipamento de Controle de Tensão j para [VCS] de (4.19)...........89

Tabela 4.5 - Dados do Sistema-Teste de 7 Barras....................................... 91

Tabela 4.6 - Dados do Sistema-Teste de 7 Barras / Região B ..........................93 
Tabela 4.7 - Equipamentos de Controle de Tensão com Efeito Oposto na Tensão da Barra Controlada i para [VCS] de (4.30)...

Tabela 4.8 - Barras de Tensão Controlada Afetadas de Forma Oposta pelo Equipamento de Controle de Tensão j para [VCS] de (4.30) .94

Tabela $4.9-\Delta V$ Após Aumento de 0,01 pu na Tensão da Barra 2 .....................96

Tabela $4.10-\triangle C$ Após Aumento de 0,01 pu na Tensão da Barra 2 ...................96

Tabela 4.11 - $\Delta V$ Após Redução de 0,01 pu na Barra 2 .................................97

Tabela $4.12-\Delta C$ Após Redução de 0,01 pu na Tensão da Barra 2 ....................98

Tabela $4.13-\Delta V$ Após Aumento de 0,01 pu na Tensão da Barra 2 - Região B ..98

Tabela $4.14-\Delta C$ Após Aumento de 0,01 pu na Barra 2 - Região B ....................99

Tabela 4.15 - $\Delta V$ Após Redução de 0,01 pu na Barra 2 - Região B ..................100

Tabela 4.16 - $\Delta$ C Após Redução de 0,01 pu na Barra 2 - Região B ...................100

Tabela 4.17 - Características do Sistema S-SE-CO Brasileiro ..........................101

Tabela 4.18 - Resultados da Análise Modal para a Matriz de (4.17) ..................110

Tabela 4.19 - Resultados da Análise Modal para a Matriz de (4.19) ..................112

Tabela 4.20 - Resultados da Análise Modal para a Matriz de (4.28) ..................113

Tabela 4.21 - Resultados da Análise Modal para a Matriz de (4.30) ..................114

Tabela 4.22 - Dados do Sistema-Teste de 12 Barras....................................116

Tabela 4.23 - Resultados da Análise Modal para a Matriz de (4.57) ................117

Tabela 4.24 - Autovalores do Sistema-Teste de 12 Barras para Diversos Níveis de Carga

Tabela 4.25 - FPs Após Aumento da Carga do Sistema-Teste de 12 Barras ....120

Tabela 4.26 - Dados do Sistema-Teste de 12 Barras

Tabela 4.27 - Relatório da Avaliação das Condições de Estabilidade de Tensão do Sistema-Teste de 12 Barras

Tabela 4.28 - Equipamentos de Controle de Tensão com Efeito Oposto na Tensão da Barra Controlada i para [VCS] de (4.58) 122

Tabela 4.29 - Barras de Tensão Controlada Afetadas de Forma Oposta pelo Equipamento de Controle de Tensão j para [VCS] de (4.58) .......123

Tabela 4.30 - Resultados da Análise Modal para a Matriz de (4.58) .123

Tabela 4.31 - Relatório da Avaliação das Condições de Estabilidade de Tensão do Sistema-Teste New England 126

Tabela 4.32 - Resultados da Análise Modal Aplicada à Matriz [VCS] de (4.59) .129

Tabela 4.33 - Variação nas Tensões Internas dos Geradores Após Aumento da Tensão da Barra 34 em 0,01 pu.

Tabela 4.34 - Variação nas Tensões Internas dos Geradores Após Redução da Tensão da Barra 39 em 0,01 pu 
Tabela 4.35 - Resultados da Análise Modal Aplicada à Matriz [VCS] de (4.60) .133

Tabela 4.36 - Resultados da Análise Modal Aplicada à Matriz [VCS] de (4.61) .137

Tabela 4.37 - Variação nas Tensões Internas dos Geradores e nos Tapes dos LTCs Após Aumento da Tensão da Barra 2 em 0,008 pu 140

Tabela 4.38 - Variação nas Tensões Internas dos Geradores e nos Tapes dos LTCs Após Aumento da Tensão da Barra 12 em 0,017 pu..........140

Tabela 4.39 - Relatório da Avaliação das Condições de Estabilidade de Tensão do Sistema-Teste SAGE 35 Barras

Tabela 4.40 - Resultados da Análise Modal Aplicada à Matriz [VCS] de (4.62) .146

Tabela 4.41 - Variação nas Tensões Controladas por Geradores e Reatores Após Aumento da Susceptância da Barra 8 em 0,5 pu

Tabela 4.42 - Variação nas Tensões Controladas por Geradores e Reatores Após Aumento da Susceptância das Barras 8 e 9 em 1,0 pu ......149

Tabela 4.43 - Relatório da Avaliação das Condições de Estabilidade de Tensão do S-SE Brasileiro às 18 horas

Tabela 4.44 - Resultados da Análise Modal Aplicada à Matriz [VCS] de (4.63) .153

Tabela 4.45 - Relatório da Avaliação das Condições de Estabilidade de Tensão do S-SE Brasileiro às 15h

Tabela 4.46 - Resultados da Análise Modal Aplicada à Matriz [VCS] de (4.64) .155

Tabela 5.1 - Geração e Carga / Caso-Base ...................................................164

Tabela 5.2 - Geração e Carga / Ponto de Operação Obtido ...............................165

Tabela 5.3 - Dados do Sistema-Teste de 12 Barras.......................................169

Tabela 5.4 - Relatório de Segurança de Tensão para o Sistema-Teste IEEE-24 Barras no Ponto de Operação Inicial

Tabela 5.5 - Perfil de Tensão do Sistema-Teste IEEE-24 Barras Antes e Após Reforço

Tabela 5.6 - Sistema-Teste IEEE 24 Barras / Ajuste em $\Delta C$ Passo a Passo .....175

Tabela 5.7 - Relatório de Segurança de Tensão para o Sistema-Teste IEEE-24 Barras no Ponto de Operação Obtido Após Mudança no Perfil de Tensão.

Tabela 5.8 - Autovalores e FPs para a Matriz [VCS] de (5.8)

Tabela 5.9 - Resultados da Análise Modal Aplicada à Matriz [VCS] de (5.6) .....179 


\section{Lista de Abreviaturas e Siglas}

CEPEL Centro de Pesquisas de Energia Elétrica

CE Compensador Estático

CS Compensador Síncrono

CSC Controlled Series Compensator

FC Fixed Capacitor

II Índice de Influência

IEEE Institute of Electrical and Electronics Engineers

FRJ Somatório dos fluxos de potência ativa chegando à Área Rio

FP Fator de Participação

LTC Load Tap Changing

M Margem de potência

Mo Margem de potência no caso-base

$M_{1} \quad$ Margem de potência após o evento em análise

OLTC On-load tap changing

P Potência Ativa

Q Potência Reativa

SAGE Sistema Aberto de Gerenciamento de Energia

SPA Solução Parcial de Autovalores

S-SE Sul/ Sudeste

S-SE-CO Sul / Sudeste e Centro Oeste

SVC Static Var Compensator

TRC Thyristor controlled reactor

VS Voltage Stability

VSA Voltage Stability Assessment

WSCC Western Systems Coordnating Council

$\theta \quad$ Ângulo da Tensão

V Módulo da Tensão

$\Delta \mathrm{P} \quad$ Variação incremental de potência ativa

$\Delta \mathrm{Q} \quad$ Variação incremental de potência reativa

$\Delta \theta \quad$ Variação incremental do ângulo da tensão

$\Delta \mathrm{V} \quad$ Variação incremental do módulo da tensão

$\Delta \mathrm{Eg} \quad$ Variação incremental do módulo da tensão interna do gerador 
[J] Matriz Jacobiana

[A] Submatriz da matriz Jacobiana expandida

[B] Submatriz da matriz Jacobiana expandida

[C] Submatriz da matriz Jacobiana expandida

[D] Submatriz da matriz Jacobiana expandida

[D] Matriz obtida de uma redução da matriz Jacobiana expandida

[VCS] Voltage control sensitivity matrix

$\partial \mathrm{P} / \partial \theta \quad$ Derivada parcial da potência ativa em relação ao ângulo da tensão

$\partial \mathrm{P} / \partial \mathrm{V}$ Derivada parcial da potência ativa em relação ao módulo da tensão

$\partial \mathrm{P} / \partial \mathrm{t} \quad$ Derivada parcial da potência ativa em relação ao tape de um LTC

$\partial \mathrm{P} / \partial \mathrm{Eg}$ Derivada parcial da potência ativa em relação ao módulo da tensão interna de um gerador ou compensador síncrono

$\partial \mathrm{P} / \partial \mathrm{b}_{\text {sh }}$ Derivada parcial da potência ativa em relação a susceptância em derivação

$\partial \mathrm{P} / \partial \mathrm{B}_{\mathrm{S} v \mathrm{c}}$ Derivada parcial da potência ativa em relação a admitância em derivação de um SVC

$\partial \mathrm{P} / \partial \alpha \quad$ Derivada parcial da potência ativa em relação ao ângulo de disparo de disparo dos tiristores de um SVC

$\partial \mathrm{Q} / \partial \theta \quad$ Derivada parcial da potência reativa em relação ao módulo da tensão

$\partial \mathrm{Q} / \partial \mathrm{V}$ Derivada parcial da potência reativa em relação ao módulo da tensão

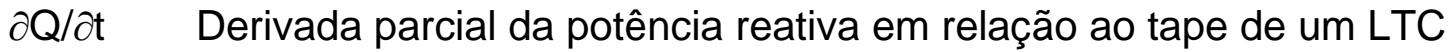

$\partial \mathrm{Q} / \partial \mathrm{Eg}$ Derivada parcial da potência reativa em relação ao módulo da tensão interna de um gerador ou compensador síncrono

$\partial \mathrm{Q} / \partial \mathrm{b}_{\mathrm{sh}}$ Derivada parcial da potência reativa em relação a susceptância em derivação

$\partial \mathrm{Q} / \partial \mathrm{B}_{\mathrm{Svc}}$ Derivada parcial da potência reativa em relação a admitância em derivação de um SVC

$\partial \mathrm{Q} / \partial \alpha \quad$ Derivada parcial da potência reativa em relação ao ângulo de disparo de disparo dos tiristores de um SVC

$\mathrm{S}_{\mathrm{i}} \quad$ Injeção de potência aparente na barra i

$\mathrm{S}_{\mathrm{m}} \quad$ Injeção máxima de potência aparente na barra i

$\nabla P \quad$ Gradiente do fluxo de potência ativa

$\nabla Q \quad$ Gradiente do fluxo de potência reativa

$\beta \quad$ Ângulo entre os gradientes dos fluxos de potência ativa e reativa

Eg Módulo da tensão interna do gerador

Z Módulo da impedância 
$b_{\text {sh }} \quad$ Susceptância em derivação

$\alpha \quad$ Ângulo da impedância

$\lambda$ Autovalor

$\phi \quad$ Autovetor à direita

$\psi \quad$ Autovetor à esquerda 
"Pouco conhecimento faz que as criaturas se sintam orgulhosas. Muito conhecimento, que se sintam humildes. É assim que as espigas sem grãos erguem desdenhosamente a cabeça para o céu, enquanto que as cheias as baixam para a terra, sua mãe." 